\title{
Genetic variation at ERBB3/IKZF4 and sexual dimorphism in epitope spreading in single autoantibody-positive relatives
}

\author{
Julie Vandewalle ${ }^{1}$ (D) Bart J. Van der Auwera ${ }^{1}$ (D) $\cdot$ Henna Amin $^{1}$ (D) $\cdot$ Erik Quartier $^{1}$ (D) Aster K. Desouter $^{1,2}$ (D) \\ Sylvie Tenoutasse $^{3}$ (D) Pieter Gillard ${ }^{1,2,4}$ (D) Christophe De Block $^{5}$ (D) Bart Keymeulen $^{1,2}$ (D) Frans K. Gorus ${ }^{1,2}$ (D) \\ Mark Van de Casteele ${ }^{1}$ (D) the Belgian Diabetes Registry
}

Received: 15 March 2021 / Accepted: 9 June 2021 / Published online: 26 August 2021

(C) The Author(s) 2021

\begin{abstract}
Aims/hypothesis We examined whether the non-HLA susceptibility locus ERBB3/IKZF4 influences progression of type 1 diabetes stage specifically according to sex.

Methods SNPs of ERBB3 (rs2292239 T/G) and IKZF4 (rs1701704 G/T) were screened by allelic discrimination quantitative PCR assay in first-degree relatives of type 1 diabetes patients who had developed at least one circulating autoantibody. The effect of $E R B B 3 / I K Z F 4$ genotypes and sex, on the progression of single autoantibody positivity to multiple autoantibody positivity and from multiple autoantibody positivity to diabetes, was studied by Kaplan-Meier analysis and multivariate Cox regression.

Results In the cohort of autoantibody-positive first-degree relatives, the risk allele frequencies for ERBB3 rs2292239 (T) and IKZF4 rs1701704 (G) were increased. There was a significant male excess at the stage of multiple autoantibody positivity $(p=$ 0.021). In Kaplan-Meier survival analysis, progression from single to multiple antibody positivity was delayed in female participants with genotype ERBB3 GG $(p=0.018$, vs ERBB3 TG+TT) or IKZF4 TT ( $p=0.023$, vs IKZF4 GT+GG), but not in male participants. In multivariate Cox regression models, the interaction effects between female sex and $E R B B 3 \mathrm{GG}(p=$ $0.012 ; \mathrm{HR}=0.305$ [95\% CI 0.120, 0.773]) or between female sex and IKZF4 TT ( $p=0.011 ; \mathrm{HR}=0.329$ [95\% CI 0.140, 0.777]) emerged as potential determinants of delayed progression to multiple autoantibodies. The progression from multiple autoantibody positivity to type 1 diabetes appeared not to be influenced by ERBB3/IKZF4.

Conclusions/interpretation In siblings and offspring of type 1 diabetes patients, polymorphism in region ERBB3/IKZF4 may affect disease progression at the level of epitope spreading in female individuals. Our findings suggest that interaction between sex and ERBB3/IKZF4 may contribute to the post-pubertal male excess in type 1 diabetes.
\end{abstract}

Keywords Beta cell function $\cdot E R B B 3 \cdot$ Gender $\cdot I K Z F 4 \cdot$ Prediabetes $\cdot$ Prediction $\cdot$ Sex $\cdot$ SNP $\cdot$ Type 1 diabetes

Members of the Belgian Diabetes Registry who enrolled patients for this study are listed in the Electronic supplementary material (ESM)

Mark Van de Casteele

mvdcaste@vub.be

1 Diabetes Research Center, Vrije Universiteit Brussel (VUB), Brussels, Belgium

2 Diabetes Clinic, Universitair Ziekenhuis Brussel (UZ Brussel), Brussels, Belgium

3 Diabetology Clinic, Hôpital Universitaire des Enfants Reine Fabiola (HUDERF), Université Libre De Bruxelles, Brussels, Belgium

4 Department of Endocrinology, University Hospitals Leuven, Leuven, Belgium

5 Department of Endocrinology, Diabetology and Metabolism, Universitair Ziekenhuis Antwerpen, Edegem, Belgium

\author{
Abbreviations \\ autoAb Autoantibody \\ autoAb ${ }^{+}$Autoantibody-positive \\ BDR Belgian Diabetes Registry \\ FDR First-degree relative \\ IAA Insulin autoantibodies \\ IA-2A Insulinoma-associated antigen-2 autoantibodies \\ ZnT8A Zinc transporter 8 autoantibodies
}

\section{Introduction}

Type 1 diabetes is characterised by an immune-mediated destruction of pancreatic beta cells leading to insulin deficiency. Unlike 


\section{Research in context}

\section{What is already known about this subject?}

- The incidence of type 1 diabetes sharply declines after puberty in female individuals, but not in male individuals, generating a significant male excess in adult-onset disease

- This is also reflected in a higher prevalence of autoantibodies in male as compared with female first-degree relatives

- Genetic polymorphism (rs2292239/rs1701704) at the ERBB3/IKZF4 locus contributes to both risk and progression of type 1 diabetes, while the ERBB3 gene is involved in regulation of hormone-dependent cellular processes

\section{What is the key question?}

- Does polymorphism at ERBB3/KZF4 impact progression of islet autoimmunity in first-degree relatives in a sex- and disease stage-specific way?

\section{What are the new findings?}

- Absence of risk alleles (genotypes ERBB3 GG or IKZF4 TT) slows progression from single to multiple autoantibody positivity in female individuals, but not in male individuals, coinciding with a male preponderance in multiple, but not in single, autoantibody-positive relatives

- Female sex interacts with ERBB3 GG or IKZF4 TT carriership to predict slower progression of islet autoimmunity, independently from previously identified predictors

- ERBB3- or IKZF4-inferred risk does not affect progression from multiple autoantibody positivity to clinical onset in our dataset

\section{How might this impact on clinical practice in the foreseeable future?}

- If confirmed by other independent cohort studies, these results may have implications for the selection and treatment of participants in future clinical intervention studies

other autoimmune diseases, it exhibits a strong male bias for diagnosis after age 15 years due to a steep post-pubertal drop in incidence in female individuals only [1,2]. This is also reflected in a higher prevalence of islet autoantibodies (autoAbs) in male than in female first-degree relatives (FDRs), especially after age 10 years [3]. It has been suggested that this male excess may relate to positive direct and indirect effects of 17betaOH-oestradiol (E2) and oestrogen receptors on beta cell formation, function and survival $[4,5]$.

In children at genetic or familial risk followed from birth, male sex was reported to confer a higher risk of developing islet autoimmunity by age 6 years [6], but to date there are no indications of more rapid disease progression in autoantibody-positive (auto $\mathrm{Ab}^{+}$) male individuals. If anything, multiple auto $\mathrm{Ab}^{+}$girls were reported to develop clinical onset more rapidly than boys [7]. However, the striking age-dependent disease heterogeneity [8] also warrants investigations in older risk groups which generate the majority of individuals eligible for prevention trials, as well as the majority of new-onset patients [1].

In our cohort of persistently autoAb ${ }^{+}$FDRs sex was not an independent determinant of progression from single to multiple autoAb positivity, or from multiple positivity to clinical onset, in multivariate analysis $[9,10]$. However, non-HLA polymorphisms may exert a stage-specific impact on disease progression in at-risk (sub)groups in time-to-event analysis [11-13]. We wondered whether confirmed non-HLA susceptibility genes encoding proteins expressed in beta cells and implicated in cell survival and proliferation may contribute to sexual dimorphism in progression of subclinical islet autoimmunity. In this context ERBB3 (which encodes erb-B2 receptor tyrosine kinase 3 [ERBB3]) emerged as a prime candidate to be investigated, as ERBB3 is expressed in various cell types including beta cells [14], and can modulate expression and transcriptional activity of oestrogen receptors [15]. Genetic variation in the ERBB3/IKZF4 region was repeatedly associated with type 1 diabetes [14] and used to improve prediction of islet autoimmunity and disease progression [11, 13]. We hypothesised that ERBB3, or nearby genes, may exert stage-related and/or sex-related effects on the progression of asymptomatic disease in a population at increased familial risk. We selected SNPs rs2292239 and rs1701704, located near $E R B B 3 / I K Z F 4$, to investigate this in a cohort of auto $\mathrm{Ab}^{+}$ FDRs followed by the Belgian Diabetes Registry (BDR) [9, 10].

\section{Methods}

Participants The BDR identified and followed 462 persistently auto $\mathrm{Ab}^{+}$siblings and offspring (under 40 years of age at first positivity) of type 1 diabetes patients between March 1989 and December 2015 among a group of 7029 FDRs enrolled after 
informed consent from the relatives or their legal representative $[9,10]$. Progression of the relatives through the different stages of subclinical autoimmunity is visualised in Electronic supplementary material (ESM) Fig. 1 (see ESM Methods: Participants, for further details).

Analytical methods AutoAbs against insulin (IAA), GAD65 (GADA), insulinoma-associated antigen-2 (IA-2A) and zinc transporter 8 (ZnT8A) were previously measured by liquidphase radiobinding assays and $H L A-D Q$ and $H L A-A$ genotypes by allele-specific oligonucleotide hybridisation [9, 10]. ERBB3 rs2292239 and IKZF4 rs1701704 were genotyped by allelic discrimination using TaqMan SNP genotyping assays C_15967467_10 and C_8340619_10, respectively (cat no. 4351379, Applied Biosystems, Foster City, CA, USA), on a QuantStudio 12 K Flex Real-Time PCR System (Applied Biosystems) (see ESM Methods: Analytical methods, for further details).

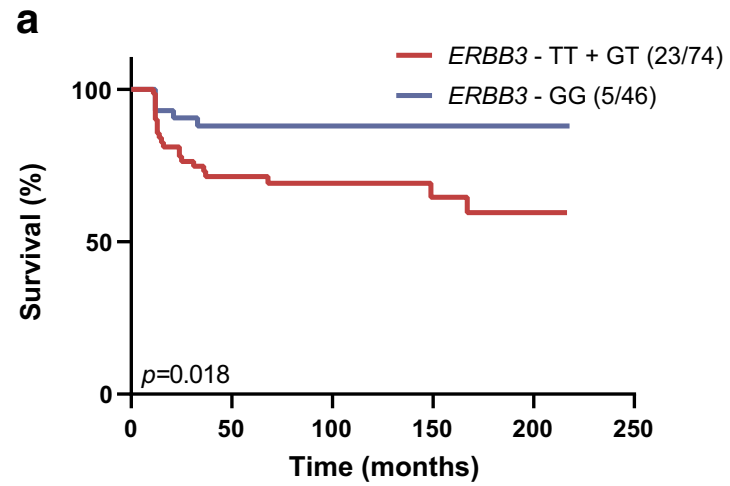

Number at risk
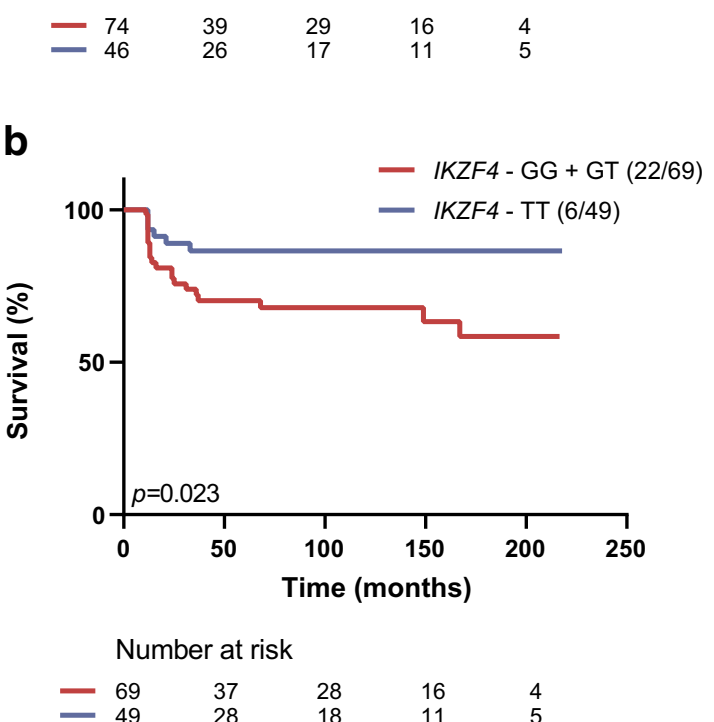

Fig. 1 Sex-specific effect of ERBB3 and IKZF4 on the development of multiple autoAbs. Kaplan-Meier survival plots for conversion from single to multiple autoAb positivity according to presence (red line) or absence (blue line) of at least one risk allele for $E R B B 3$-rs2292239 (a, c) or IKZF4-rs $1701704(\mathbf{b}, \mathbf{d})$ in either female $(\mathbf{a}, \mathbf{b})$ or male $(\mathbf{c}, \mathbf{d})$ FDRs.
Statistical analyses Statistical differences between groups were analysed with the Pearson $\chi^{2}$ test for categorical variables and with the Kruskal-Wallis test for continuous variables. KaplanMeier survival analysis with logrank test and multivariate Cox regression analysis were used to assess progression from single to multiple auto $\mathrm{Ab}$ positivity and from multiple autoAb positivity to diabetes for different SNP genotypes, according to sex. Twotailed statistical tests were performed and $p$ values $<0.05$ were considered significant (see ESM Methods: Statistical analyses, for further details).

\section{Results}

ERBB3 rs2292239 and IKZF4 rs1701704 genotypes Both SNPs had call rates $>98 \%$ within our autoAb ${ }^{+}$FDR cohort (ESM Fig. 1). The minor and major allelic frequencies of both SNPs differed significantly from those in the European
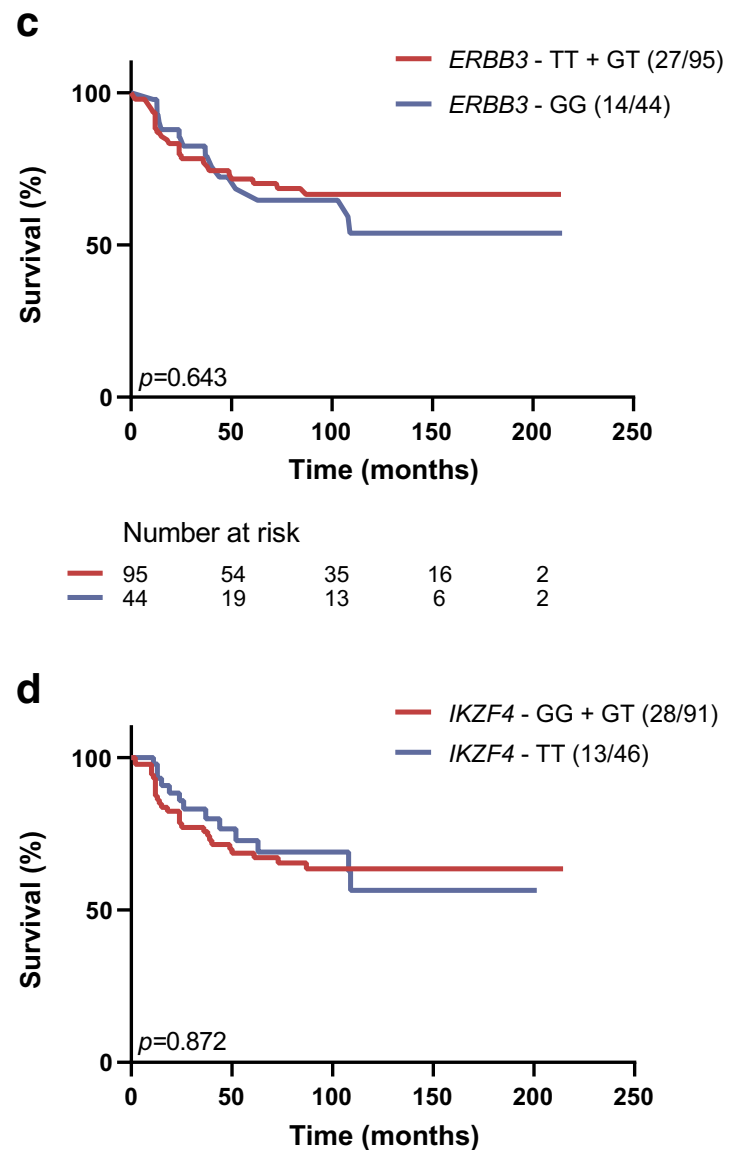

Number at risk

$\begin{array}{rrrcr}-91 & 50 & 35 & 17 & 3 \\ -\quad 46 & 20 & 13 & 5 & 1\end{array}$

For each arm the genotype and number (events/cases) are indicated above the graph. The numbers of individuals at risk are indicated below the $x$ axis. Significant effects $(p<0.05)$ were observed for ERBB3 and IKZF4 in female participants, but not in male participants 
population in the 1000 Genomes Project $(p<0.01$; ESM Table 1, ESM Methods), with an increased prevalence of the ERBB $3 \mathrm{~T}$ and IKZF4 $\mathrm{G}$ risk alleles in the cohort. Genotype distributions for both SNPs did not deviate significantly from the Hardy-Weinberg equilibrium ( $p>0.05$; ESM Table 1). General characteristics of the study population are presented per ERBB3/IKZF4 genotype and subclinical stage (ESM Tables 2,3). There was a significant male excess at the stage of multiple autoAb positivity ( $p=0.021$; ESM Table 3$)$, but not at the stage of single autoAb positivity $(p=0.38$; ESM Table 2).

ERBB3/IKZF4 SNPs and progression from single to multiple autoAb positivity Both SNPs affected epitope spreading according to sex in Kaplan-Meier analysis. Progression from single to multiple autoAb positivity was slowed in female participants without $E R B B 3$ risk (T) alleles ( $p=0.018$ vs female participants carrying $\geq 1 \mathrm{~T}$ allele; Fig. 1a), and in female participants without IKZF4 risk (G) alleles ( $p=0.023$ vs presence of $\geq 1 \mathrm{G}$ allele; Fig. 1b). No such delay was observed in male participants (Fig. 1c,d).

The suggested interaction between female sex and ERBB3 GG or IKZF4 TT genotype for delaying epitope spreading was further examined by multivariate Cox regression analysis. Stepwise conditional forward models were built separately for ERBB3 and IKZF4 (Table 1). In both models, the absence of
IAA as first autoAb $(p<0.05)$, older age at first autoAb positivity $(p<0.001)$, absence of the $H L A-D Q 2 / D Q 8$ high-risk genotype $(p<0.001)$ and presence of the HLA-A*24 allele $(p<0.02)$ delayed the development of multiple autoAbs (Table 1), in line with previous findings $[9,10]$. In both the model for $E R B B 3$ and for IKZF4, being female or carrying the low-risk genotype (GG and TT, respectively) did not impact progression to multiple autoAb positivity when considering both variables separately (Table 1). However, the interaction effect between ERBB3 GG and female sex ( $p=0.012$; HR = 0.305 [95\% CI $0.120,0.773]$ ) or between IKZF4 TT and female $\operatorname{sex}(p=0.011 ; \mathrm{HR}=0.329$ [95\% CI $0.140,0.777])$ significantly delayed epitope spreading (Table 1 ). No interaction between $E R B B 3$ or IKZF4 and the previously reported independent determinants of epitope spreading [10] reached significance in multivariate analysis (Table 1).

ERBB3/IKZF4 SNPs and progression from multiple autoAb positivity to clinical onset Cox regression models built for $E R B B 3$ or IKZF4 confirmed previously reported independent risk factors $\left(H L A-A^{*} 24\right.$, IA- $2 \mathrm{~A}^{+}$or $\mathrm{ZnT} 8 \mathrm{~A}^{+}$, younger age) for accelerated progression from multiple auto $A b$ positivity to clinical onset $[9,10]$. However, progression was not influenced by ERBB3 or IKZF4 genotype, be it alone or in interaction with sex or established predictors (ESM Table 4).
Table 1 Cox regression analysis of progression from single auto $\mathrm{Ab}$ positivity to multiple autoAb positivity in FDRs

\begin{tabular}{|c|c|c|c|c|}
\hline \multirow[t]{2}{*}{ Variable } & \multicolumn{2}{|c|}{ Model ERBB3 } & \multicolumn{2}{|c|}{ Model IKZF4 } \\
\hline & $p$ & $\operatorname{HR}(95 \% \mathrm{CI})$ & $p$ & HR $(95 \% \mathrm{CI})$ \\
\hline Age first autoAb ${ }^{+}$ & $<0.001$ & $0.913(0.881,0.947)$ & $<0.001$ & $0.914(0.882,0.947)$ \\
\hline Non-IAA $\left(0 / 1^{\mathrm{a}}\right)$ & 0.037 & $0.576(0.343,0.967)$ & 0.043 & $0.585(0.348,0.983)$ \\
\hline $\operatorname{Sex}\left(0 / 1^{b}\right)$ & NM & & NM & \\
\hline$H L A-A * 24\left(0 / 1^{\mathrm{a}}\right)$ & 0.017 & $0.405(0.193,0.851)$ & 0.011 & $0.381(0.181,0.802)$ \\
\hline Non- $(H L A-D Q 2 / D Q 8)\left(0 / 1^{\mathrm{a}}\right)$ & $<0.001$ & $0.332(0.193,0.570)$ & $<0.001$ & $0.349(0.203,0.600)$ \\
\hline$E R B B 3-\mathrm{GG}\left(0 / 1^{\mathrm{a}}\right)$ & NM & & - & \\
\hline$E R B B 3-\mathrm{GG} \times$ age first auto $\mathrm{Ab}^{+}$ & NM & & - & \\
\hline ERBB3-GG $\times$ non-IAA & NM & & - & \\
\hline$E R B B 3-\mathrm{GG} \times \mathrm{sex}$ & 0.012 & $0.305(0.120,0.773)$ & - & \\
\hline$E R B B 3-\mathrm{GG} \times H L A-A * 24$ & $\mathrm{NM}$ & & - & \\
\hline$E R B B 3-\mathrm{GG} \times$ non- $(H L A-D Q 2 / D Q 8)$ & NM & & - & \\
\hline$I K Z F 4-\mathrm{TT}\left(0 / 1^{\mathrm{a}}\right)$ & - & & NM & \\
\hline$I K Z F 4-\mathrm{TT} \times$ age first autoAb ${ }^{+}$ & - & & NM & \\
\hline$I K Z F 4-\mathrm{TT} \times$ non-IAA & - & & NM & \\
\hline$I K Z F 4-\mathrm{TT} \times \operatorname{sex}$ & - & & 0.011 & $0.329(0.140,0.777)$ \\
\hline$I K Z F 4-\mathrm{TT} \times H L A-A * 24$ & - & & NM & \\
\hline IKZF4-TT $\times$ non- $(H L A-D Q 2 / D Q 8)$ & - & & NM & \\
\hline \multicolumn{5}{|c|}{ Models built by multivariate analysis included either $E R B B 3$ or $I K Z F 4$} \\
\hline \multicolumn{5}{|c|}{${ }^{\mathrm{a}} 0 / 1:$ no/yes } \\
\hline \multicolumn{5}{|l|}{${ }^{\mathrm{b}} 0 / 1$ : male/female } \\
\hline
\end{tabular}




\section{Discussion}

Our main finding is that the GG genotype of rs2292239 in the $E R B B 3$ gene slows progression of subclinical islet autoimmunity in FDRs, but that this effect is restricted to female individuals and to the phase of epitope spreading. Similar results were obtained for the closely linked TT genotype of rs1701704 located near IKZF4. This sexual dimorphism in protective action is independent from already known variables associated with slower epitope spreading (older age, presence of $H L A-A * 24$, absence of IAA and/or $H L A-D Q 2 / D Q 8)$ [10]. It appears specific for the ERBB3/IKZF4 region as it was not observed for established predictors of progression. The increased prevalence of ERBB3/IKZF4 risk alleles in our auto $\mathrm{Ab}^{+} \mathrm{FDR}$ population further suggests that these alleles contribute to the disease risk. The data are also in agreement with the reported male excess in auto $\mathrm{Ab}^{+}$individuals [3] and suggest that ERBB3/IKZF4 may contribute to the male excess observed in FDRs with multiple autoAbs (ESM Table 3). However, these findings require confirmation in independent cohorts.

To date, most studies in auto $\mathrm{Ab}^{+}$individuals have been following young children, often from birth after preselection for HLA class II-inferred risk and/or prior islet cell antibody (ICA) testing [11-13]. Our approach, to include also auto $\mathrm{Ab}^{+}$ adolescents and young adults, is deemed a strength in the context of the present report because autoAbs can appear at any age while most patients develop clinical symptoms in adulthood with a strong post-pubertal male bias $[1,9]$.

Oestrogens and oestrogen receptors are known to impact beta cell function and formation, as well as immune cell responses, in a sex-dependent way [4, 5]. Since ERBB3 has been shown to control oestrogen receptor expression and function [15], one may speculate functional interactions between $E R B B 3$ and oestrogen receptors in beta and/or immune cells to underlie the sex-specific effect of $E R B B 3 /$ $I K Z F 4$. It has previously been reported that ERBB3/IKZF4 polymorphisms associate with higher risk of developing (multiple) autoAbs, and with accelerated progression to clinical onset after seroconversion to single autoAb positivity [12]. Our results suggest that this reported acceleration may rather be interpreted as a selective delay in epitope spreading in ERBB3 GG/IKZF4 TT female individuals. Together with the reported lower proneness to auto $\mathrm{Ab}$ positivity in female vs male individuals [6], this delay may contribute to the selective post-pubertal drop in incidence in female individuals [1], but requires confirmation in multiple auto $\mathrm{Ab}^{+}$individuals identified in the general population. Given the low number of FDRs followed from the stage of first autoAb positivity to clinical onset, the impact of the ERBB3 genotype on overall time to clinical onset could not be accurately determined in this study.

In conclusion, we report that interaction of $E R B B 3 / I K Z F 4$ and female sex appears to delay the progression from 1 to $\geq 2$
autoAbs and may possibly contribute to lower disease incidence in female individuals, which needs confirmation in independent cohorts of at-risk individuals.

Supplementary Information The online version of this article (https://doi. org/10.1007/s00125-021-05546-9) contains peer-reviewed but unedited supplementary material.

Acknowledgements We thank C. Groven for researching data (BDR, UZ Brussel, Belgium). The authors sincerely thank all BDR members who have contributed to the recruitment of relatives for this study. They are listed in the ESM.

Data availability The datasets generated and/or analysed during the present study are not openly available as they were derived from pseudonymised data and samples collected by the Belgian Diabetes Registry, a controlled access repository of sensitive human data. They can be made available upon reasonable request to co-author B. Keymeulen, president of the Belgian Diabetes Registry.

Funding The study was supported by grant WFWG18-18 of the 'Wetenschappelijk Fonds Willy Gepts' of the University Hospital Brussels (UZ Brussel), and by the Vrije Universiteit Brussel (Strategic Research Programmes [SRP] Growth 43 and SRP Spearhead 55).

Author's relationships and activities The authors declare that there are no relationships or activities that might bias, or be perceived to bias, their work.

Contribution statement JV, MVdC, HA and FKG contributed to the study design, data acquisition, analysis and interpretation of data, and drafting of the article. BJVdA, AKD and EQ contributed to data acquisition. ST, PG, CDB and BK contributed to the study design. All the authors critically revised the manuscript and approved the version to be published. MVdC is the guarantor of this work and, as such, had full access to all the data in the study and takes responsibility for the integrity of the data and the accuracy of the data analysis.

Open Access This article is licensed under a Creative Commons Attribution 4.0 International License, which permits use, sharing, adaptation, distribution and reproduction in any medium or format, as long as you give appropriate credit to the original author(s) and the source, provide a link to the Creative Commons licence, and indicate if changes were made. The images or other third party material in this article are included in the article's Creative Commons licence, unless indicated otherwise in a credit line to the material. If material is not included in the article's Creative Commons licence and your intended use is not permitted by statutory regulation or exceeds the permitted use, you will need to obtain permission directly from the copyright holder. To view a copy of this licence, visit http://creativecommons.org/licenses/by/4.0/.

\section{References}

1. Vandewalle CL, Coeckelberghs MI, De Leeuw IH et al (1997) Epidemiology, clinical aspects, and biology of IDDM patients under age 40 years. Comparison of data from Antwerp with 
complete ascertainment with data from Belgium with $40 \%$ ascertainment. The Belgian Diabetes Registry. Diabetes Care 20(10): 1556-1561. https://doi.org/10.2337/diacare.20.10.1556

2. Gale EA, Gillespie KM (2001) Diabetes and gender. Diabetologia 44(1):3-15. https://doi.org/10.1007/s001250051573

3. Williams AJ, Bingley PJ, Moore WP, Gale EA, ENDIT Screening Group, European Nicotinamide Diabetes Intervention Trial (2002) Islet autoantibodies, nationality and gender: a multinational screening study in first-degree relatives of patients with Type I diabetes. Diabetologia 45(2):217-223. https://doi.org/10.1007/s00125-0010749-6

4. Liu S, Mauvais-Jarvis F (2010) Minireview: estrogenic protection of beta-cell failure in metabolic diseases. Endocrinology 151(3): 859-864. https://doi.org/10.1210/en.2009-1107

5. Yuchi Y, Cai Y, Legein B et al (2015) Estrogen receptor alpha regulates beta-cell formation during pancreas development and following injury. Diabetes 64(9):3218-3228. https://doi.org/10. 2337/db14-1798

6. Krischer JP, Lynch KF, Lernmark A et al (2017) Genetic and environmental interactions modify the risk of diabetes-related autoimmunity by 6 years of age: The TEDDY study. Diabetes Care 40(9): 1194-1202. https://doi.org/10.2337/dc17-0238

7. Krischer JP, Liu X, Lernmark A et al (2017) The influence of type 1 diabetes genetic susceptibility regions, age, sex, and family history on the progression from multiple autoantibodies to type 1 diabetes: A TEDDY study report. Diabetes 66(12):3122-3129. https://doi. org $/ 10.2337 / \mathrm{db} 17-0261$

8. Gorus FK (1997) Diabetes registries and early biological markers of insulin-dependent diabetes mellitus. Belgian Diabetes Registry. Diabetes Metab Rev 13(4):247-274. https://doi.org/10.1002/(sici) 1099-0895(199712)13:4<247::aid-dmr196>3.0.co;2-v

9. Gorus FK, Balti EV, Messaaoui A et al (2017) Twenty-year progression rate to clinical onset according to autoantibody profile, age, and HLA-DQ genotype in a registry-based group of children and adults with a first-degree relative with type 1 diabetes. Diabetes Care 40(8):1065-1072. https://doi.org/10.2337/dc16-2228

10. Balke EM, Balti EV, Van der Auwera B et al (2018) Accelerated progression to type 1 diabetes in the presence of HLA-A*24 and $\mathrm{B} * 18$ is restricted to multiple islet autoantibody-positive individuals with distinct HLA-DQ and autoantibody risk profiles. Diabetes Care 41(5):1076-1083. https://doi.org/10.2337/dc17-2462

11. Winkler C, Krumsiek J, Lempainen J et al (2012) A strategy for combining minor genetic susceptibility genes to improve prediction of disease in type 1 diabetes. Genes Immun 13(7):549-555. https:// doi.org/10.1038/gene.2012.36

12. Lempainen J, Laine AP, Hammais A et al (2015) Non-HLA gene effects on the disease process of type 1 diabetes: From HLA susceptibility to overt disease. J Autoimmun 61:45-53. https://doi.org/10. 1016/j.jaut.2015.05.005

13. Torn C, Hadley D, Lee HS et al (2015) Role of type 1 diabetesassociated SNPs on risk of autoantibody positivity in the TEDDY study. Diabetes 64(5):1818-1829. https://doi.org/10.2337/db141497

14. Kaur S, Mirza AH, Brorsson CA et al (2016) The genetic and regulatory architecture of ERBB3-type 1 diabetes susceptibility locus. Mol Cell Endocrinol 419:83-91. https://doi.org/10.1016/j. mce.2015.10.002

15. St-Laurent V, Sanchez M, Charbonneau C, Tremblay A (2005) Selective hormone-dependent repression of estrogen receptor beta by a p38-activated ErbB2/ErbB3 pathway. J Steroid Biochem Mol Biol 94(1-3):23-37. https://doi.org/10.1016/j.jsbmb.2005.02.001

Publisher's note Springer Nature remains neutral with regard to jurisdictional claims in published maps and institutional affiliations. 